\title{
Humic Acid Can Enhance the Mineralization of Phenanthrene Sorbed on Biochars
}

Meng Zhang ${ }^{\dagger \dagger}$, Xiaofang Shen ${ }^{\dagger}$, Haiyun Zhang ${ }^{\dagger}$, David Werner ${ }^{\zeta}$, Bin Wang ${ }^{\eta}$, Yu Yang ${ }^{\varphi}$, Shu Tao Xilong Wang ${ }^{\dagger}, *$

$\dagger$ Laboratory for Earth Surface Processes, College of Urban and Environmental Sciences, Peking University, Beijing 100871, China

$\$$ Co-Innovation Center for Sustainable Forestry in Southern China, College of Biology and the Environment, Nanjing Forestry University, Nanjing 210037, Jiangsu Province, China

${ }^{\zeta}$ School of Civil Engineering and Geosciences, Newcastle University, Newcastle upon Tyne NE1 7RU, U.K.

$\eta^{\eta}$ Department of Epidemiology and Biostatistics, School of Public Health, Peking University, Beijing 100191, China

${ }^{\varphi}$ Department of Civil and Environmental Engineering, University of Nevada, Reno, Nevada 89557, United States

\section{Supporting Information}

Number of pages: 10

Number of tables: 4 


\section{Biochar treatment}

The biochars were washed with $0.1 \mathrm{M} \mathrm{HCl}$ to remove carbonates, soluble salts, and potassium compounds, and decrease their $\mathrm{pH}$ values, followed by rinsing with DI water until neutral $\mathrm{pH}$ was reached. The samples were then dried in an oven at $105^{\circ} \mathrm{C}$, gently milled to pass through a $250 \mu \mathrm{m}$ sieve, and stored for further use.

\section{Solid-state ${ }^{13} \mathrm{C}$ NMR running parameters}

Solid-state ${ }^{13} \mathrm{C}$ NMR spectra of biochars were obtained with a Bruker Advance III $400 \mathrm{MHz}$ NMR spectrometer operated at a ${ }^{13} \mathrm{C}$ frequency of $100 \mathrm{MHz}$ and at a magic-angle-spinning rate of $5 \mathrm{kHz}$ through a $7 \mathrm{~mm} \mathrm{H}-\mathrm{X}$ MAS probe. Samples were packed in a zirconium rotor (OD $=7 \mathrm{~mm})$ with a KelF cap. A standard ramp-CP pulse program was used for spectra scanning and the running parameters were as follows: contact time of $2 \mathrm{~ms}$, recycle delay time of $1 \mathrm{~s}$, line broadening of $50 \mathrm{~Hz}$, and number of scans 5000 .

\section{Methods used to determine SA and porosity.}

Prior to $\mathrm{N}_{2}$ adsorption, samples were outgassed at $105^{\circ} \mathrm{C}$ for over $16 \mathrm{~h}$. The $\mathrm{SAs}$ of samples were derived from the multipoint sorption isotherms of $\mathrm{N}_{2}$ at $77 \mathrm{~K}$ using the Brunauer-Emmett-Teller method with relative pressure $P / P_{0}$ from 0.05 to 0.3 . The micropore volumes of samples were determined using the Dubinin-Radushkevich model with $P / P_{0} \leq 0.05$ and their meso- and macropore volumes were calculated from the desorption isotherms employing the Barrett-Joyner-Halenda model. 
Table S1. Bulk elemental composition, surface area, porosity, and solid-state ${ }^{13} \mathrm{C}$

NMR data of biochars

Table S2. Bulk elemental composition, solid-state ${ }^{13} \mathrm{C}$ NMR data and molecular weight of HA. SA: surface area; $\mathrm{V}_{\text {mic }}$ : micropore volume; $\mathrm{V}_{\text {mes }}+\mathrm{V}_{\text {mac }}$ : sum of the meso- and macropore volumes. R700, S700, P700, M700, and B700 represent the biochars obtained from manures of rabbit, sheep, and pig, and straws of maize and bean at 700 ${ }^{\circ} \mathrm{C}$, respectively.

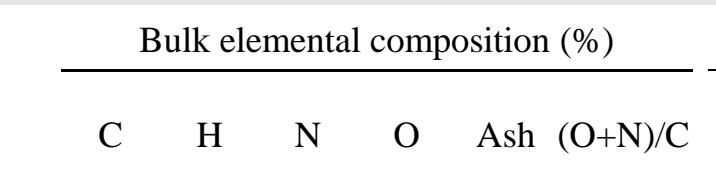
Chemical shift (ppm) and carbon assignment

Molecular $\begin{array}{llllll}0-50 & 50-109 & 109-145 & 145-163 & 163-190 & 190-220\end{array}$ Weight alkyl O-alkyl aromatic phenolic carboxyl carbonyl (Dalton)

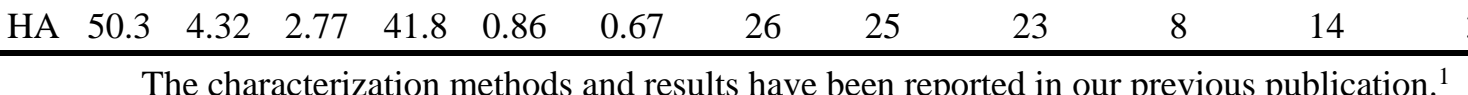

70

71

72

73

74 
Table S3. Rates and extents of mineralization within $480 \mathrm{~h}$ of PHE sorbed to various biochars for different HA concentrations.

First-order model parameters maximum mineralization $480 \mathrm{~h}$ mineralized

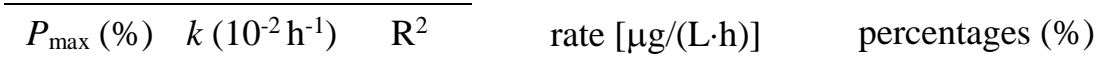

Rabbit manure $+700^{\circ} \mathrm{C}$

$\begin{array}{lccccc}\text { R700 } & 13.7 \pm 0.4 & 5.6 \pm 1.0 & 0.987 & 8.4 \pm 0.5 & 13.8 \pm 0.2 \\ \text { R700+20mg/L HA } & 16.1 \pm 1.6 & 6.0 \pm 0.9 & 0.996 & 10.7 \pm 1.6 & 16.3 \pm 1.4 \\ \text { R700+200mg/L HA } & 16.9 \pm 0.5 & 9.6 \pm 0.1 & 0.995 & 17.8 \pm 1.5 & 17.0 \pm 0.3 \\ \text { Sheep manure+700 }{ }^{\circ} \mathrm{C} & & & & & \\ \text { S700 } & 14.9 \pm 0.3 & 8.4 \pm 0.9 & 0.984 & 13.8 \pm 1.5 & 15.0 \pm 0.5 \\ \text { S700+20mg/L HA } & 21.6 \pm 3.9 & 8.6 \pm 1.9 & 0.985 & 20.4 \pm 1.8 & 21.7 \pm 1.3 \\ \text { S700+200mg/L HA } & 21.2 \pm 1.6 & 12.9 \pm 0.5 & 0.995 & 30.1 \pm 2.4 & 21.3 \pm 1.6 \\ \text { Pig manure+700 }{ }^{\circ} \mathrm{C} & & & & & \\ \text { P700 } & 22.4 \pm 5.2 & 8.5 \pm 3.9 & 0.990 & 21.0 \pm 5.0 & 22.6 \pm 1.5 \\ \text { P700+20mg/L HA } & 39.0 \pm 3.7 & 9.4 \pm 0.6 & 0.983 & 40.2 \pm 4.5 & 39.3 \pm 2.3 \\ \text { P700+200mg/L HA } & 39.5 \pm 6.2 & 12.3 \pm 0.1 & 0.984 & 53.3 \pm 2.2 & 39.9 \pm 3.6 \\ \text { Maize stalk+700 }{ }^{\circ} \mathrm{C} & & & & & \\ \text { M700 } & 22.6 \pm 2.7 & 5.5 \pm 0.2 & 0.992 & 13.7 \pm 1.6 & 22.5 \pm 1.2 \\ \text { M700+20mg/L HA } & 43.1 \pm 0.8 & 5.9 \pm 0.2 & 0.984 & 28.0 \pm 1.7 & 42.9 \pm 1.8 \\ \text { M700+200mg/L HA } & 45.1 \pm 0.8 & 9.0 \pm 0.3 & 0.994 & 44.9 \pm 4.7 & 45.0 \pm 0.6 \\ \text { Bean straw+700 }{ }^{\circ} \mathrm{C} & & & & & \\ \text { B700 } & 28.1 \pm 0.5 & 7.5 \pm 0.9 & 0.987 & 23.3 \pm 1.9 & 27.7 \pm 1.5 \\ \text { B700+20mg/L HA } & 43.0 \pm 2.0 & 6.8 \pm 0.9 & 0.992 & 32.0 \pm 3.5 & 42.4 \pm 1.1 \\ \text { B700+200mg/L HA } & 47.9 \pm 0.4 & 8.4 \pm 0.6 & 0.995 & 44.3 \pm 4.5 & 47.3 \pm 0.4 \\ \text { Without biochar } & & & & & \\ \text { Without HA } & 56.1 \pm 0.8 & 12.6 \pm 0.0 & 0.980 & 78.0 \pm 9.1 & 55.9 \pm 1.8 \\ \text { 20 mg/L HA } & 78.3 \pm 2.1 & 13.8 \pm 0.9 & 0.977 & 118.8 \pm 12.4 & 78.5 \pm 2.2 \\ \text { 200 mg/L HA } & 89.8 \pm 1.8 & 14.9 \pm 0.3 & 0.992 & 147.1 \pm 13.1 & 90.0 \pm 1.8\end{array}$

$P_{\max }(\%)$ and $k\left(10^{-2} \mathrm{~h}^{-1}\right)$ are the maximum mineralized percentage and the first-order rate constant for mineralization of PHE sorbed to biochars as fitted by first-order equation (3) in the main text; The maximum mineralization rate is calculated as $P_{\max } / 100 \% \cdot k \cdot[\mathrm{PHE}](\mu \mathrm{g} / \mathrm{L} \cdot \mathrm{h})$, where $[\mathrm{PHE}]$ is the total concentration of PHE initially present in the experimental suspension $(1.10 \mathrm{mg} / \mathrm{L}=1100 \mu \mathrm{g} / \mathrm{L})$. Here, errors refer to standard errors of three replicate experiments. 
Table S4. Kinetic parameters, rates and amounts for desorption of PHE from various biochars as obtained with Tenax extraction in the presence of two different concentrations of HA

\begin{tabular}{|c|c|c|c|c|c|c|c|c|c|c|c|}
\hline \multicolumn{7}{|c|}{ Three-phase model parameters } & \multicolumn{4}{|c|}{ Desorption rate $[\mu \mathrm{g} /(\mathrm{L} \cdot \mathrm{h})]$} & \multirow{2}{*}{$\begin{array}{r}\text { Desorption } \\
\text { amount at } \\
480 \mathrm{~h}(\%)\end{array}$} \\
\hline $\begin{array}{c}F_{\text {rapid }} \\
(\%)\end{array}$ & $\begin{array}{c}k_{\text {rapid }} \\
\left(10^{-1} h^{-1}\right)\end{array}$ & $\begin{array}{c}F_{\text {slow }} \\
(\%)\end{array}$ & $\begin{array}{c}k_{\text {slow }} \\
\left(10^{-2} \mathrm{~h}^{-1}\right)\end{array}$ & $\begin{array}{c}F_{\text {vslow }} \\
(\%)\end{array}$ & $\begin{array}{c}k_{\text {vslow }} \\
\left(10^{-5} \mathrm{~h}^{-1}\right)\end{array}$ & $\mathrm{R}^{2}$ & $v_{\text {rapid }}$ & $v_{\text {slow }}$ & $v_{\text {vslow }}$ & $v_{\text {average }}$ & \\
\hline
\end{tabular}

\begin{tabular}{|c|c|c|c|c|c|c|c|c|c|c|c|}
\hline R700 & $5.7 \pm 0.7$ & $3.10 \pm 0.63$ & $21.1 \pm 0.4$ & $1.40 \pm 0.12$ & $53.2 \pm 2.3$ & $6.9 \pm 0.4$ & 0.996 & $87.6 \pm 9.0$ & $3.25 \pm 0.150 .04 \pm 0.00$ & $90.9 \pm 8.3$ & $48.5 \pm 0.9$ \\
\hline R700+20mg/L HA & $26.7 \pm 1.3$ & $3.16 \pm 0.62$ & $22.5 \pm 0.7$ & $1.24 \pm 0.06$ & $50.8 \pm 0.4$ & $10.5 \pm 1.2$ & 0.995 & $92.8 \pm 17.0$ & $3.06 \pm 0.080 .06 \pm 0.00$ & $95.9 \pm 15.7$ & $51.0 \pm 0.5$ \\
\hline $700+200 \mathrm{mg} / \mathrm{L} \mathrm{HA}$ & $27.2 \pm 0.8$ & $3.14 \pm 0.64$ & $23.5 \pm 0.1$ & $1.26 \pm 0.13$ & $49.3 \pm 2.5$ & $8.9 \pm 1.3$ & 0.995 & $94.1 \pm 11.3$ & $3.25 \pm 0.030 .05 \pm 0.01$ & $97.4+9.0$ & $52.7 \pm 0.3$ \\
\hline 700 & $26.5 \pm 0.5$ & $3.04 \pm 0.12$ & $22.1 \pm 0.6$ & $1.35 \pm 0.01$ & $51.5 \pm 1.0$ & $8.2 \pm 0.2$ & 0.991 & $88.5 \pm 1.4$ & $3.28 \pm 0.020 .05 \pm 0.00$ & $91.8 \pm 0.9$ & $50.4 \pm 0.6$ \\
\hline 700+20mg/L HA & $27.8 \pm 0.0$ & $3.08 \pm 0.02$ & $24.0 \pm 0.8$ & $1.19 \pm 0.10$ & $48.3 \pm 0.9$ & $12.4 \pm 1.8$ & 0.995 & $94.1 \pm 0.0$ & $3.13 \pm 0.180 .07 \pm 0.00$ & $97.3 \pm 0.0$ & $53.8 \pm 2.0$ \\
\hline S700+200mg/L HA & $28.6 \pm 0.7$ & $3.05 \pm 0.85$ & $25.3 \pm 0.6$ & $1.20 \pm 0.26$ & $46.1 \pm 2.0$ & $11.5 \pm 1.6$ & 0.995 & $96.1 \pm 6.5$ & $3.33 \pm 0.540 .06 \pm 0.01$ & $99.5 \pm 3.7$ & $56.3 \pm 1.3$ \\
\hline P700 & $27.3 \pm 0.8$ & $2.98 \pm 0.96$ & $23.0 \pm 0.6$ & $1.30 \pm 0.22$ & $49.7 \pm 1.7$ & $9.4 \pm 1.9$ & 0.996 & $89.7 \pm 24.2$ & $3.30 \pm 0.410 .05 \pm 0.01$ & $93.1 \pm 11.6$ & $52.5 \pm 0.8$ \\
\hline P700+20mg/L HA & $28.8 \pm 0.3$ & $3.02 \pm 0.18$ & $25.4 \pm 0.6$ & $1.14 \pm 0.36$ & $45.8 \pm 0.8$ & $14.5 \pm 1.9$ & 0.995 & $95.6 \pm 4.7$ & $3.19 \pm 0.440 .07 \pm 0.00$ & $98.9 \pm 1.6$ & $56.5 \pm 1.0$ \\
\hline P700+200mg/L HA & $29.9 \pm 0.8$ & $3.00 \pm 0.21$ & $27.2 \pm 0.8$ & $1.15 \pm 0.02$ & $42.9 \pm 2.4$ & $14.6 \pm 1.9$ & 0.996 & $98.6 \pm 1.9$ & $3.45 \pm 0.050 .07 \pm 0.01$ & $102.1 \pm 0.9$ & \pm 1.6 \\
\hline 700 & $28.0 \pm 0.3$ & $2.94 \pm 0.72$ & $23.8 \pm 0.6$ & $1.27 \pm 0.38$ & $48.3 \pm 1.8$ & $10.6 \pm 1.7$ & 0.996 & $90.6 \pm 2.4$ & $3.32 \pm 0.760 .06 \pm 0.01$ & $94.0 \pm 1.9$ & $54.0 \pm 0.5$ \\
\hline M700+20mg/L HA & $29.5 \pm 0.6$ & $2.98 \pm 0.79$ & $26.2 \pm 1.0$ & $1.12 \pm 0.03$ & $44.3 \pm 0.5$ & $15.9 \pm 1.1$ & 0.995 & $96.7 \pm 14.0$ & $3.24 \pm 0.050 .08 \pm 0.00$ & \pm 13.9 & $58.0 \pm 1.4$ \\
\hline M700+200mg/L HA & $31.7 \pm 0.5$ & $2.89 \pm 0.88$ & $29.6 \pm 0.8$ & $1.10 \pm 0.03$ & $38.7 \pm 1.4$ & $19.5 \pm 3.4$ & 0.996 & $101.0 \pm 6.3$ & $3.59 \pm 0.090 .08 \pm 0.01$ & $104.7 \pm 4.4$ & $64.0 \pm 2.6$ \\
\hline B700 & $29.4 \pm 0.7$ & $2.89 \pm 0.84$ & $26.0 \pm 0.6$ & $1.23 \pm 0.02$ & $44.6 \pm 0.3$ & $11.2 \pm 0.1$ & 0.996 & $93.4 \pm 9.5$ & $3.53 \pm 0.050 .05 \pm 0.00$ & $97.0 \pm 8.4$ & $57.7 \pm 0.4$ \\
\hline B 700+20mg/L HA & $31.2 \pm 0.7$ & $2.91 \pm 0.62$ & $29.2 \pm 1.0$ & $1.10 \pm 0.13$ & $39.6 \pm 0.6$ & $17.0 \pm 2.5$ & 0.996 & $100.0 \pm 9.5$ & $3.53 \pm 0.380 .07 \pm 0.00$ & $103.6 \pm 8.9$ & $63.3 \pm 2.1$ \\
\hline B $700+200 \mathrm{mg} / \mathrm{L}$ HA & $34.2 \pm 0.8$ & $2.83 \pm 0.33$ & $33.6 \pm 1.2$ & $1.08 \pm 0.39$ & $32.2 \pm 2.1$ & $23.3 \pm 1.8$ & 0.99 & $106.4 \pm 14.5$ & $54.00 \pm 1.450 .08 \pm 0.01$ & $110.5 \pm 13.9$ & $70.5 \pm 3.0$ \\
\hline
\end{tabular}


82

83

84

85

86

87

88

89

90

91

92

93

94

95

96

97

98

99

100

101

102

103

104

105

106

107

108

109

110

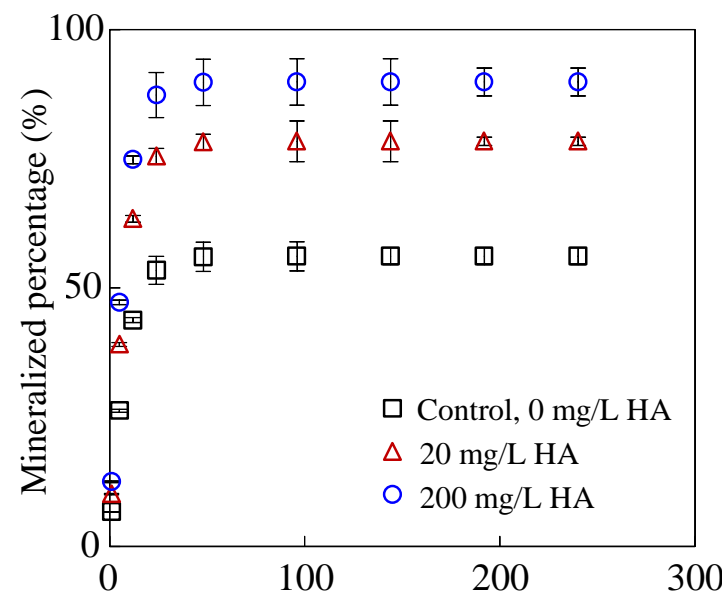

Time (h)

Figure S1. Mineralization of PHE by Mycobacterium vanbaalenii PYR-1 in the freely dissolved form at HA concentrations of $0(\square), 20(\triangle)$, and $200 \mathrm{mg} / \mathrm{L}(\circ)$. Bars represent one standard deviation $(n=3)$.

4

5

6

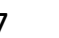

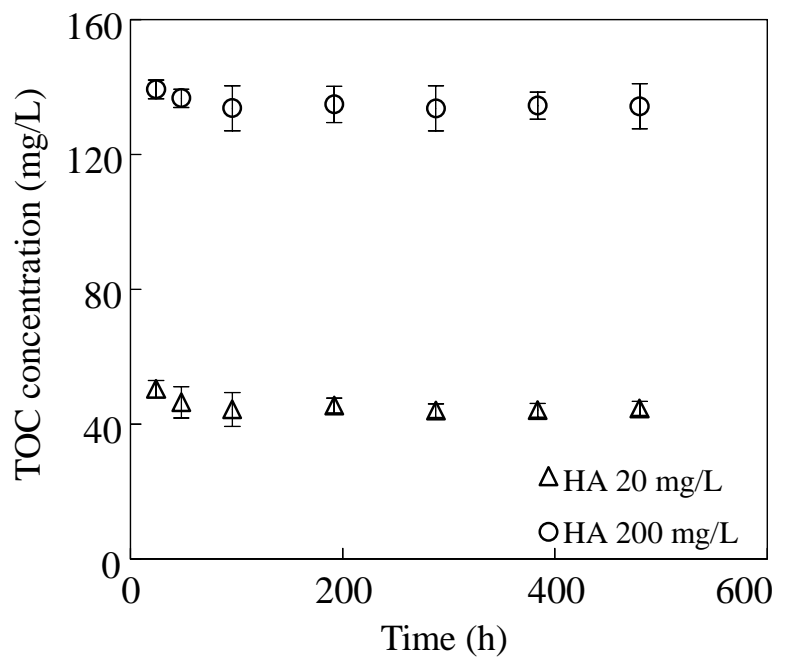

Figure S2. Total organic carbon (TOC) concentrations during the incubation of $20(\triangle)$ and $200(\circ)$ mg/L HA with Mycobacterium vanbaalenii PYR-1 at a starting OD $_{600}$ of 0.486 . Data points represent the mean values and bars represent one standard deviation from triplicate incubation flasks. 
111

112

113

114

115

116

117

118

119

120

121

122

123

124

125

126

127

128

129

130

131

132

133

134

135

136

137
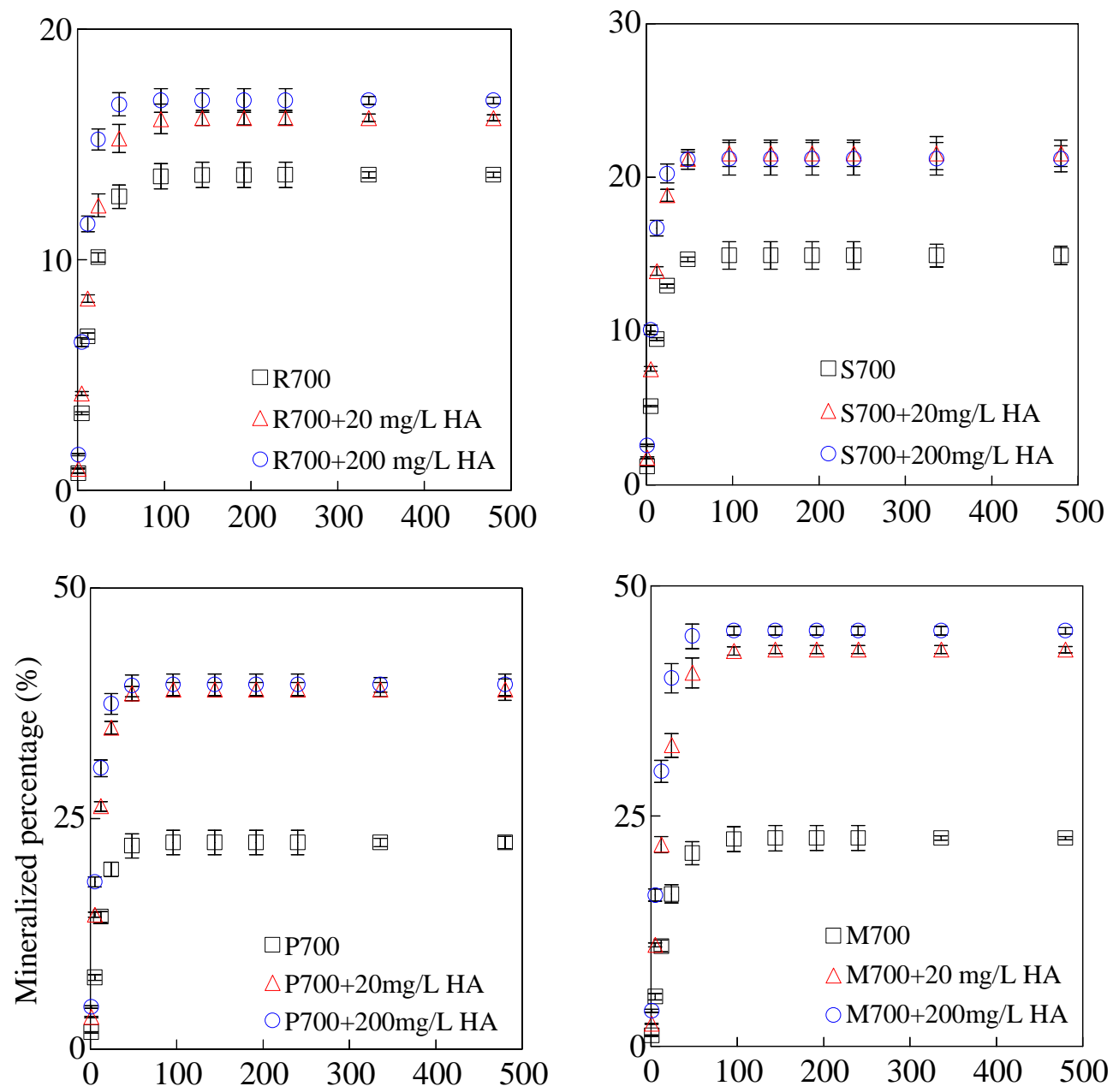

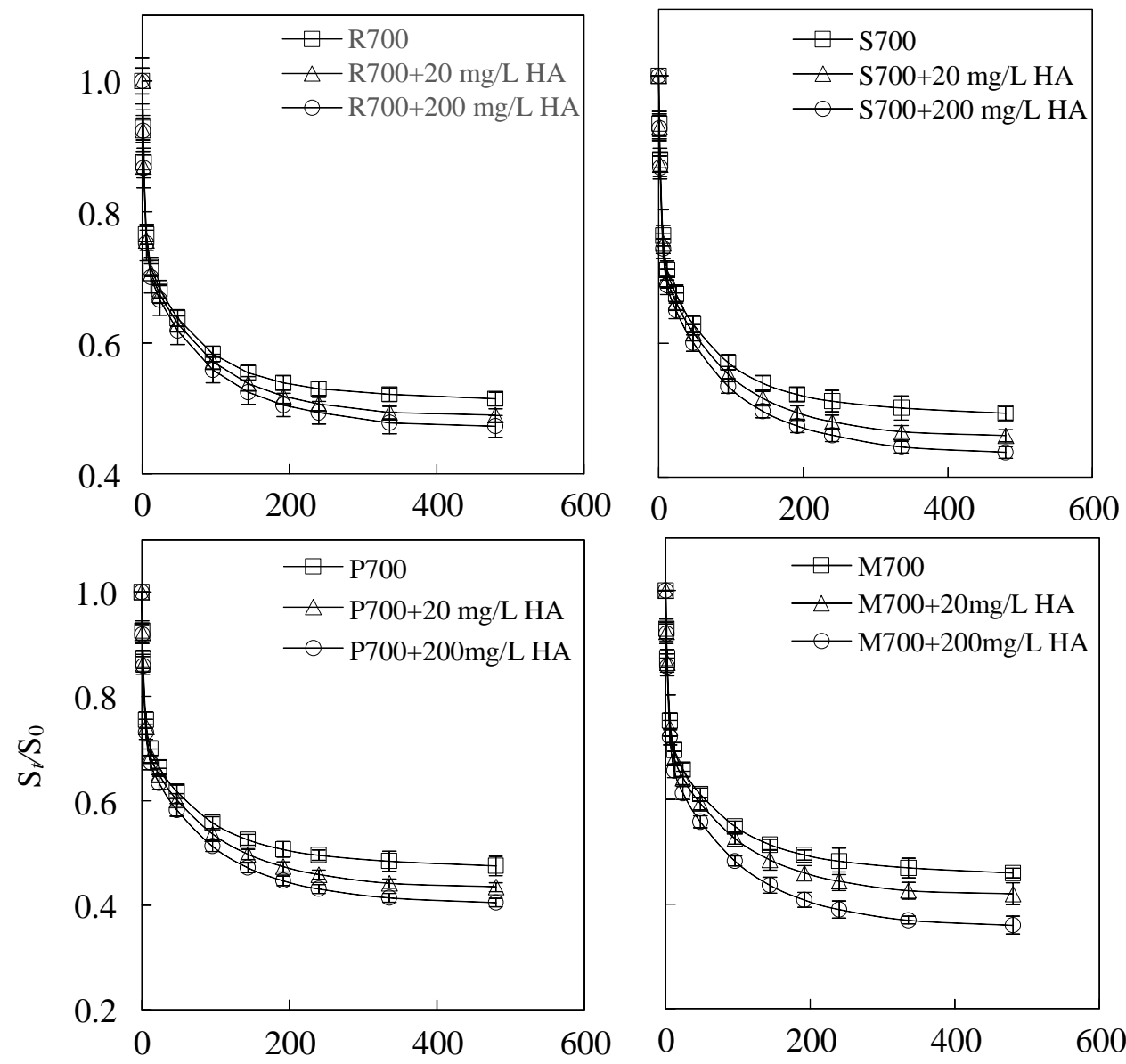

1

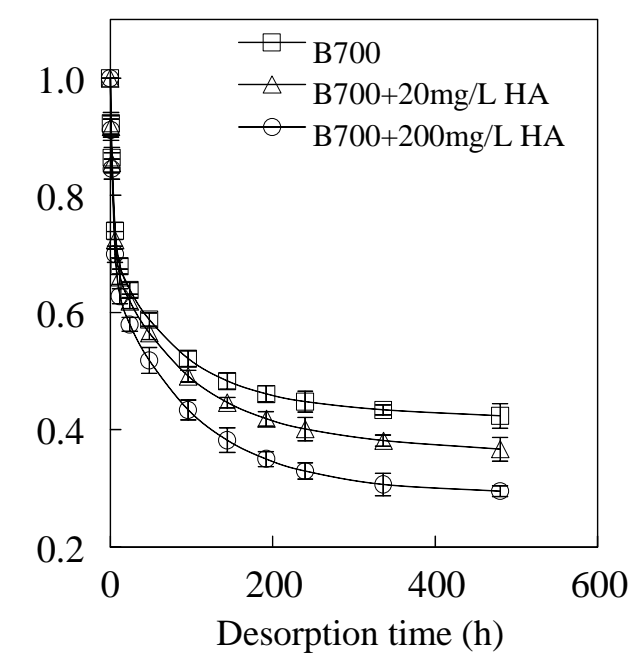

Figure S4. Abiotic desorption kinetics of PHE initially spiked on five biochars (i.e., R700, S700, P700, M700, and B700 represent the biochars obtained from manures of rabbit, sheep, and pig, and straws of maize and bean at $700^{\circ} \mathrm{C}$, respectively) in the presence of $\mathrm{HA}$ at concentrations of $0(\square), 20(\triangle)$, and $200 \mathrm{mg} / \mathrm{L}(\circ) . S_{t}$ and $S_{0}(\mathrm{ng} / \mathrm{g})$ are the amounts of PHE on biochar at desorption time $t(\mathrm{~h})$ and at the 

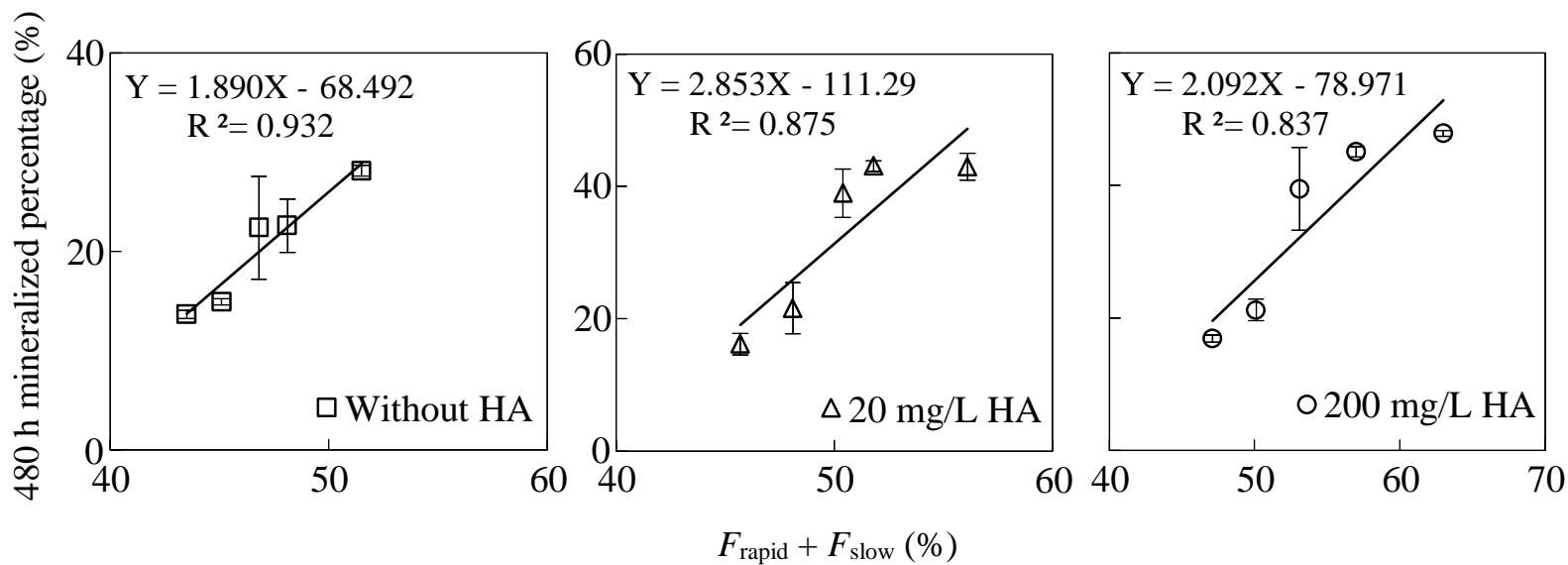

170

Figure S5. Relationship between percentages of mineralization of PHE sorbed on various biochars after $480 \mathrm{~h}$ and the sum of the rapidly and slowly desorbing fractions at different HA concentrations of 0 ( $\square$ ), $20(\triangle)$, and $200(\circ) \mathrm{mg} / \mathrm{L}$. Error bar refers to standard error of three replicate experiments..

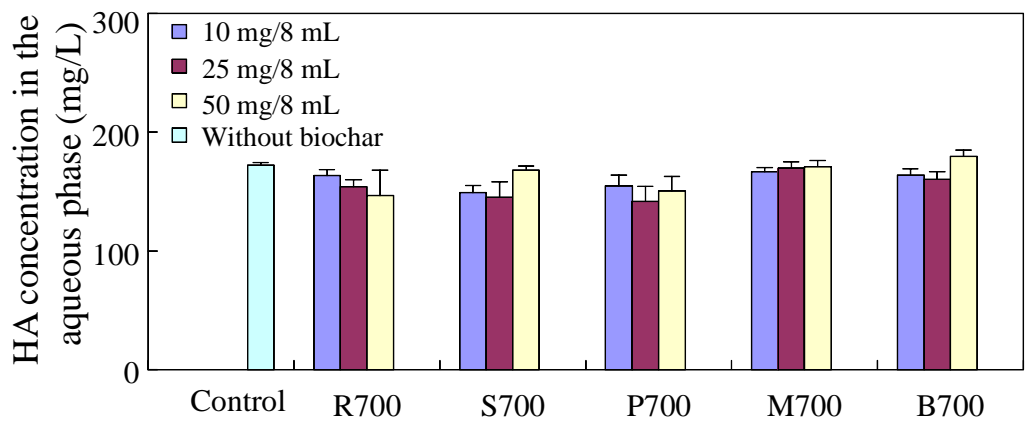

181

Figure S6. Experiments showing negligible sorption of HA on biochars: constant HA concentration equilibrated with variable amounts of biochar. Here, errors are standard deviations of three replicate experiments. Statistical comparisons were performed with ANOVA at $p=0.05$. 


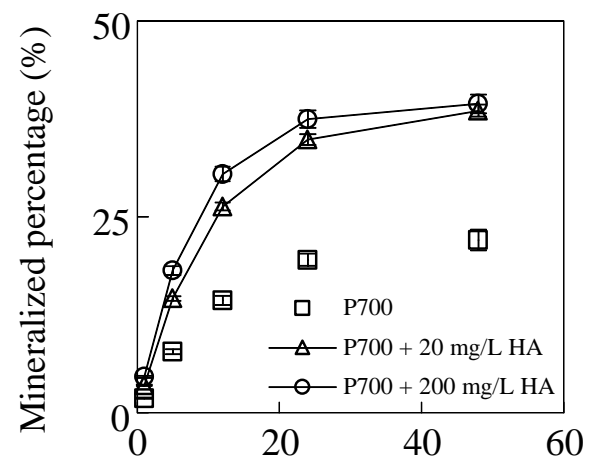

$\square \quad \mathrm{R} 700$
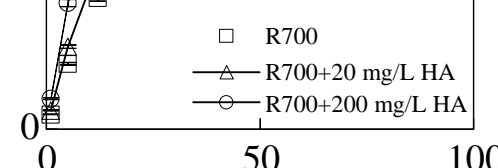

100
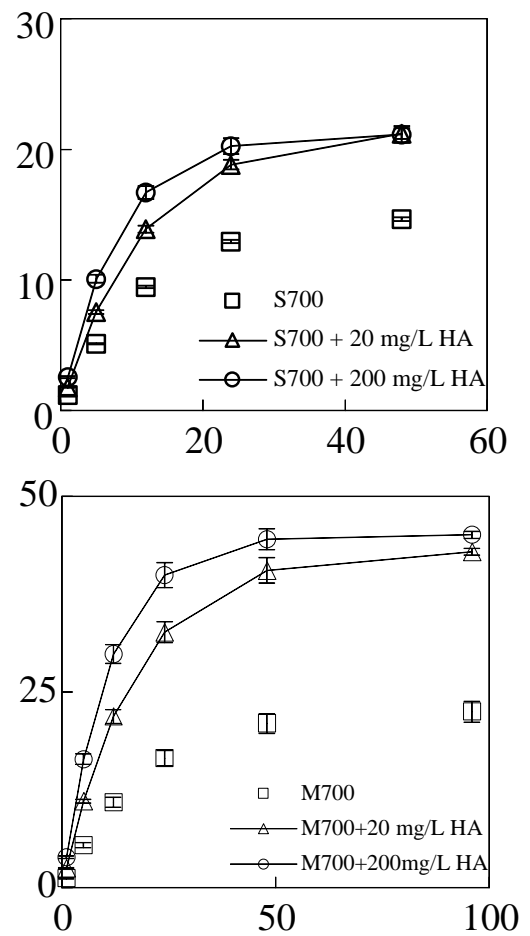

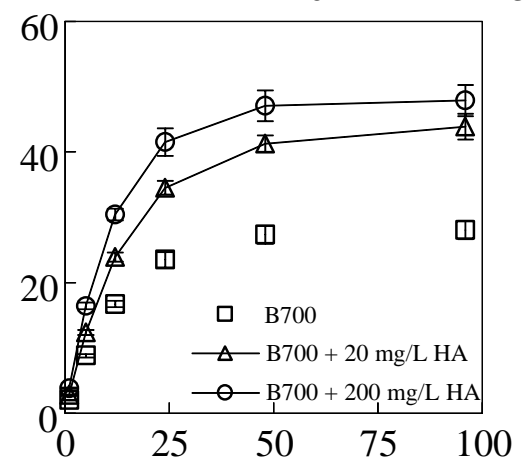

Time (h)

213

214

215

216

217

218

Figure S7. Variation of the mineralization rate of PHE sorbed to biochars at different HA concentrations of $0(\square), 20(\triangle)$, and $200(\circ) \mathrm{mg} / \mathrm{L}$. Bars represent one standard deviation $(n=3)$.

\section{Reference}

1. Zhang, M.; Shu, L.; Guo, X. Y.; Shen, X. F.; Zhang, H. Y.; Shen, G. F.; Wang, B.; Yang, Y.; Tao, S.; Wang, X. L., Impact of humic acid coating on sorption of naphthalene by biochars. Carbon 2015, 94, 946-954. 